La prévention du suicide jalonne le travail quotidien des psychiatres; les médecins de famille y sont aussi régulièrement confrontés. Mais c'est également un enjeu qui concerne toute notre société. Dans notre pays, le taux de suicide se maintient à un niveau élevé: il faut des décisions politiques à différentes échelles pour que les personnes prêtes à commettre l'irréparable trouvent plus facilement le soutien dont elles ont besoin dans les moments décisifs de leur détresse, plutôt que le moyen qui leur permettra de passer à l'acte. Le regard porté sur une Europe marquée par la crise de l'euro offre un tableau assez noir mais révèle des points surprenants.

\title{
Crise économique et santé psychique
}

Le débat sur la prévention du suicide passe régulièrement par cette réflexion fataliste selon laquelle celui qui veut se donner la mort trouvera toujours un moyen de le faire. A ce sujet, le monde scientifique tient cependant un tout autre langage: les études internationales apportent clairement la preuve que restreindre, par exemple, l'accès aux moyens de se suicider permet de réduire considérablement le taux de personnes qui passent à l'acte. La prévention du suicide existe et elle offre des possibilités qui n'ont pas été découvertes seulement depuis l'introduction des formations continues destinées aux médecins de premier recours [1] (pour la détection précoce de crises et de risque de suicide), ni depuis que la campagne de prévention «Alliance contre la dépression» fait son chemin dans toute l'Europe, ni même par le biais d'articles de presse approfondis sur le sujet. La ville de Berne a montré l'un des meilleurs exemples en la matière, en assurant notamment le pourtour de la grande terrasse de la cathédrale par des filets métalliques. En dépit des prévisions alarmistes de certains, les taux de suicides sur les ponts avoisinants n'ont pas augmenté; au contraire, ils ont également diminué. Un exemple probant que les personnes suicidaires ne veulent pas réellement mourir, mais qu'elles ne peuvent plus supporter de vivre ce qu'elles endurent.

\section{La santé psychique de la population se détériore lors de crises économiques.}

Observé dans le contexte de la crise de l'euro qui secoue toute l'Europe [2, 3], le problème du suicide prend aujourd'hui une toute autre dimension. Depuis 2007, le nombre de suicides chez les moins de 65 ans a de nouveau augmenté dans l'ensemble de l'UE. Entre janvier et mai 2011, la Grèce a enregistré une hausse du taux de suicide de $40 \%$. L'impact des mesures politiques adoptées en vue de réduire les dépenses dans le secteur de la santé est énorme: depuis 2011, 150000 personnes ont perdu leur emploi, $15 \%$ des coûts hospitaliers ont été économisés, les tarifs médicaux ont diminué de $25 \%$ et le salaire minimal de $20 \%$.

Nul n'ignore l'influence du chômage sur la santé psychique, 34\% des demandeurs d'emploi se plaignent de problèmes psychiques contre $16 \%$ des personnes qui exercent une activité stable. En Espagne, la prévalence de troubles psychiques a connu une hausse significative entre 2006 et 2010, avec l'augmentation la plus élevée pour les dépressions majeures. Les études les plus récentes mettent ces évolutions sur le compte de la perte d'un emploi et d'un chômage latent mais omniprésent.

La Finlande et la Suède font figure de bons élèves dans leur manière de gérer la crise. Malgré des chiffres du chômage en hausse, leurs taux respectifs de suicide ont continué de diminuer. Précurseurs dans le domaine social, ces deux pays ont

\section{Les pays qui assument leur responsabi- lité sociale malgré la crise affichent un taux de suicide nettement moins élevé.}

instauré par le passé des programmes intensifs de soutien social pour les demandeurs d'emploi, qu'ils ont poursuivis au plus fort de la crise économique. Là aussi, d'autres pays dotés de programmes de soutien adéquats ont été en mesure d'empêcher ou d'endiguer une croissance de leur taux de suicide.

L'Islande, un des pays heurtés de plein fouet par la crise, a fermement refusé d'imposer des économies excessives dans le domaine sanitaire et social. Les demandeurs d'emploi ont ainsi bénéficié d'un soutien actif en vue de leur réinsertion.

L'effet des décisions politiques sur la santé de la population n'a rien de nouveau. C'est à nous, le corps médical et les organisations de la santé, d'être vigilant, de porter du crédit à ce lien de cause à effet et - comme un ceterum censeo - d'en rappeler au monde politique les conséquences pour la population, pour nos patientes et pour nos patients. Au regard des statistiques actuelles en matière de suicide en Suisse, nous avons encore beaucoup de travail à faire, principalement dans la prévention chez les personnes âgées.

Barbara Weil, responsable du département Promotion de la santé et prévention

Références

1 1989: Wolfgang Rutz; J Wålinder; G Eberhard; G Holmberg; A L von Knorring; L von Knorring; B Wistedt; A Aberg-Wistedt. An educational program on depressive disorders for general practitioners on Gotland: background and evaluation. Acta psychiatrica Scandinavica 1989;79(1):19-26.

2 Lancet (Published Online) March 27, 2013, Marina Karanikolos, Philipa Mladovsky, Jonathan Cylus, Sarah Thomson, Sanjay Basu, David Stuckler, Johan P Mackenbach, Martin: Financial crisis, austerity, and health in Europe

3 Lancet 2013; 381: 1125-34, Published Online, March 27, 2013, Johan P Mackenbach, Marina Karanikolos, Martin McKee: The unequal health of Europeans: successes and failures of policies 